Check for updates

Cite this: RSC Adv., 2017, 7, 43671

\title{
Increasing local field by interfacial coupling in nanobowl arrays $\uparrow$
}

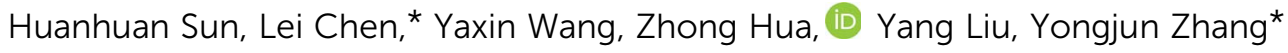 \\ and Jinghai Yang
}

An increased local field is crucial to create hotspots when applied in detections, which usually means the fabrication of nanostructure arrays with strong electromagnetic couplings. In this study, ordered monolayer $\mathrm{Ag}$ and $\mathrm{Ag} / \mathrm{SiO}_{2} / \mathrm{Ag}$ sandwich nanobowls arrays with enhanced local field are fabricated by the combination of nanosphere self-assembly lithography (NSL) technology and layer-by-layer (LBL) film deposition. The isolated nanobowls and nanohole-like nanostructures composed of connected nanobowls can be obtained by tuning the sputtering film thickness and polystyrene (PS) microsphere size. The isolated nanobowl arrays show high local surface plasmon resonance (LSPR) and surface plasmon resonance (SPR), which result in SERS enhancement. Compared to the Ag nanobowl arrays, the dielectric $\mathrm{SiO}_{2}$ layer in $\mathrm{Ag} / \mathrm{SiO}_{2} / \mathrm{Ag}$ sandwich nanobowls as a spacer layer enhances the SERS signals. And the new plasmon couplings between interior and exterior $\mathrm{Ag}$ layers through the thin $\mathrm{SiO}_{2}$ layer are implementated, in addition to the contributions of nanogaps in the adjacent nanobowls and the sharp edges of a single nanobowl. The finite-difference time-domain (FDTD) solution shows the field enhancement can be mainly attributed to the SPR of interparticles along the $\mathrm{Ag}-\mathrm{SiO}_{2}$ interfaces. The simulation results and experimental analysis indicate the important contribution of the $\mathrm{SiO}_{2}$ layer to the enhanced SERS effects in $\mathrm{Ag} / \mathrm{SiO}_{2} / \mathrm{Ag}$ sandwich nanobowl arrays.

Received 31st August 2017

Accepted 4th September 2017

DOI: $10.1039 / c 7 r a 09690 a$

rsc.li/rsc-advances and distribution). To overcome these problems, the various assembled nanostructures including nanorods, nutshells and nanocap periodic arrays, have been investigated for strong surface plasma coupling properties. ${ }^{13-15}$ The periodic nanostructures are usually fabricated by self-assembled techniques such as electron-beam lithography, ${ }^{16}$ electrochemical metal growth, ${ }^{17}$ nanoimprint lithography, ${ }^{18}$ nanosphere lithography (NSL) technology ${ }^{19-25}$ and so on. In these methods, combining NSL with a layer-by-layer (LBL) film deposition is a kind of high efficient method for the fabrication of nanostructure arrays, most materials including noble metal, semiconductor and dielectric all can be used. Noble metal Ag and dielectric $\mathrm{SiO}_{2}$ are most popular complex materials for SERS substrate due to strong plasma characteristic of $\mathrm{Ag}$ and insulative property of $\mathrm{SiO}_{2}$. Recently, some nanostructures, like nanofollowers, ${ }^{26}$ nanobowls, ${ }^{27}$ nanoposts, ${ }^{28}$ which consist of $\mathrm{Ag}$ and $\mathrm{SiO}_{2}$, have been fabricated as SERS substrate by the combination of NSL and physical deposition.

In this work, we fabricated the large-area ordered monolayer $\mathrm{Ag}$ nanobowl arrays and the $\mathrm{Ag} / \mathrm{SiO}_{2} / \mathrm{Ag}$ sandwich nanobowl arrays via the combination of NSL technology and LBL film deposition. The effects of the morphology and construction of the nanobowls on the SERS properties were investigated. Based on the finite-difference time-domain (FDTD) simulation, we studied the plasmon coupling behavior of the different material interfaces for the nanobowls arrays.
Key Laboratory of Functional Materials Physics and Chemistry, Ministry of Education, College of Physics, Jilin Normal University, Changchun 130103, P. R. China. E-mail: chenlei@jlnu.edu.cn; yjzhang@jlnu.edu.cn; $\quad$ Tel: $\quad+86-0434-3294566 ;$ +86-0434-3294566

$\dagger$ Electronic supplementary information (ESI) available. See DOI: 10.1039/c7ra09690a 


\section{Experimental section}

\subsection{Materials}

The mono-dispersed microsphere polystyrene (PS) suspension $(10 \% \mathrm{w} / \mathrm{v})$ was purchased from the Duke scientific corporation. Ethanol and tetrahydrofuran (THF) were obtained from commercial sources. $\mathrm{NH}_{4} \mathrm{OH}(25 \%)$ and $\mathrm{H}_{2} \mathrm{O}_{2}$ (30\%) were purchased from Sinopharm Chemical Reagent Co., Ltd. The 4mercaptopyridine (4-MPy, 96\%) was obtained from Aldrich. The purities of $\mathrm{Ag}$ and $\mathrm{SiO}_{2}$ targets both are $99.994 \%$ and purchased from Beijing TIANRY Science \& Technology Developing Center. Silicon wafers with the crystal orientation (100) were purchased from Hefei kejing materials technology Co., Ltd, the silicon wafer diameter and thickness are 4 inches and $0.52 \mathrm{~mm}$, respectively. De-ionized (DI) water $\left(18.0 \mathrm{M} \Omega \mathrm{cm}^{-1}\right)$ was used throughout the study.

\subsection{Assemble of two-dimensional (2D) PS periodic arrays}

Two-dimensional (2D) colloidal arrays consisting of PS nanospheres were prepared by nanosphere self-assembly lithography (NSL) technology on the silicon wafer. The detailed procedures were reported in our previous articles. ${ }^{24,29,30}$ The silicon wafers $(2 \mathrm{~cm} \times 2 \mathrm{~cm})$ were boiled in the mixed solution $\left(\mathrm{H}_{2} \mathrm{O}: \mathrm{H}_{2} \mathrm{O}_{2}: \mathrm{NH}_{4} \mathrm{OH}=6: 2: 1\right.$, volume ratio $)$ at $300{ }^{\circ} \mathrm{C}$ for 5 minutes, and then they were ultrasonically cleaned with DI water and ethanol for 15 minutes three times, respectively. Hydrophilic silicon wafers were prepared in sodium dodecyl sulfate solution (10\%) for $24 \mathrm{~h}$. Afterwards we dropped a droplet $(20 \mu \mathrm{L})$ of mixed solution (1:1 in volume) of ethanol and polystyrene solution onto the hydrophilic silicon wafer. Then the silicon wafers were slowly immersed into the glass vessel filled with DI water. The unordered monolayer PS films formed on the water surface. Then we dropped a small droplet $(8 \mu \mathrm{L})$ of sodium dodecyl sulfate solution (2\%) onto the water surface to impel the monolayer films into the highly ordered patterns. Finally, the monolayer PS films were picked up by the hydrophilic silicon wafer.

\subsection{Fabrication of $\mathrm{Ag}$ nanobowl with thickness $80 \mathrm{~nm}$ and $\mathrm{Ag}$ $40 \mathrm{~nm} / \mathrm{SiO}_{2} t \mathrm{~nm} / \mathrm{Ag} 40 \mathrm{~nm}(t=0,1,2,3)$ sandwich-structured nanobowl arrays}

The 2D PS templates were etched by plasma cleaning for different time. Ag films were deposited on the etched PS templates in magnetron sputtering system (ATC 1800-F, USA AJA). The trilaminar films $\mathrm{Ag} 40 \mathrm{~nm} / \mathrm{SiO}_{2} t \mathrm{~nm} / \mathrm{Ag} 40 \mathrm{~nm}$ with different thickness $\mathrm{SiO}_{2}$ spacer layer $(t=0,1,2$, and 3$)$ were prepared by sputtering $\mathrm{Ag}$ target and $\mathrm{SiO}_{2}$ target in turn. The $40 \mathrm{~nm} \mathrm{Ag}$ film was firstly sputtered on the top of ncp PSCP templates, then the $\mathrm{SiO}_{2}$ films with different thickness $(t=0,1,2$, and 3) were sputtered on Ag film, respectively. Finally the $40 \mathrm{~nm} \mathrm{Ag}$ film was sputtered on $\mathrm{SiO}_{2}$ film. The base pressure is $2 \times 10^{-6} \mathrm{~Pa}$ and the reaction pressure is $0.6 \mathrm{~Pa}$ during sputtering film. The sputtering power and deposition rate of $\mathrm{Ag}$ target are $21.6 \mathrm{~W}$ and $0.059 \mathrm{~nm} \mathrm{~s}^{-1}$, the sputtering power and deposition rate of $\mathrm{SiO}_{2}$ target are $100 \mathrm{~W}$ and $0.0054 \mathrm{~nm} \mathrm{~s}^{-1}$, respectively. The distance between the target and substrate is $10 \mathrm{~cm}$. Then the PS nanospheres were removed by immersing the samples into THF solution. In the end, the sputtered films are transferred to a new Si wafer.

\subsection{Characterization of substrates}

The morphologies of nanobowl arrays were investigated by scanning electron microscope (SEM, $15 \mathrm{kV}$, JEOL 6500F) and transmission electron microscope (TEM, 200 keV, JEM$2100 \mathrm{HR}$ ). The samples were characterized via X-ray diffraction (XRD, Japan Rigaku D/max-ga X-ray diffractometer, $\lambda=0.15406$ $\mathrm{nm}$ ), using CuK $\alpha$ operated at $40 \mathrm{kV}$ and $200 \mathrm{~mA}$. Before detecting the SERS samples, we generally adjust the peak of silicon wafer at $520.7 \mathrm{~cm}^{-1}$ to calibrate the spectrograph, and avoid the experimental error. For Raman spectral examination, 4-mercaptopyridine (4-MPy) solution with concentration of $10^{-3} \mathrm{~mol} \mathrm{~L}^{-1}$ was used as the probe molecule. The $\mathrm{Ag}$ nanobowl arrays and sandwich-structured $\mathrm{Ag} 40 \mathrm{~nm} / \mathrm{SiO}_{2} t \mathrm{~nm} / \mathrm{Ag} 40 \mathrm{~nm}$ $(t=0,1,2,3)$ nanobowl arrays were immersed in the 4-MPy solution $(1 \mathrm{~mL})$ for 30 minutes, respectively, which were then washed fully with ethanol and DI water for several times to remove the unabsorbed probe molecules. The samples were dried in nitrogen to measure the SERS signals. The Raman spectra were recorded in a Renishaw Raman system equipped with a chargecoupled device (CCD) detector, a $514.5 \mathrm{~nm}$ laser with a power of $4 \mathrm{~mW}$ was focused on the sample surface, covering an area of $1 \mu \mathrm{m}$ in diameter. The time of signal acquisition was $10 \mathrm{~s}$.

\subsection{FDTD solutions}

A commercial finite-difference time-domain (FDTD) software was used for simulating the distribution of the electromagnetic field. According to the experimental results, the geometric parameters in the simulation were obtained from SEM images in order to close to the real structure. The substrates were placed in the $x-z$ plane. The infinite zone were sited with periodic boundary conditions along the $x$-axis and along the $y$-axis directions, and the perfectly matched layers (PML) along the $z$ direction. ${ }^{31}$ A plane wave polarized light of wavelength $514.5 \mathrm{~nm}$ was used along the $z$-axis direction, corresponding to the excitation wavelength of the experimental SERS measurements. The refractive index of PS colloid sphere is 1.585. To maximize field enhancement resolution, the grid size was set to $0.02 \mathrm{~nm}$, and the overall simulation time was 100 fs. The value of silver permittivity and permeability used in simulations were provided by the software material database. ${ }^{26}$

\section{Results and discussion}

\subsection{Preparation of nanobowl arrays}

The fabrication process and the SEM images for the silver nanobowl arrays are illustrated in Fig. 1. Firstly, the monolayer close-packed 2D PS templates with diameter of $200 \mathrm{~nm}$ are prepared on Si wafer by NSL method (Fig. 1a). The corresponding SEM image exhibits a hexagonal close-packed array. Then these close-packed PS arrays are etched for different time by plasma etching technique (Fig. 1b). The diameter of the PS bead decreases gradually when oxygen plasma etching goes on. 


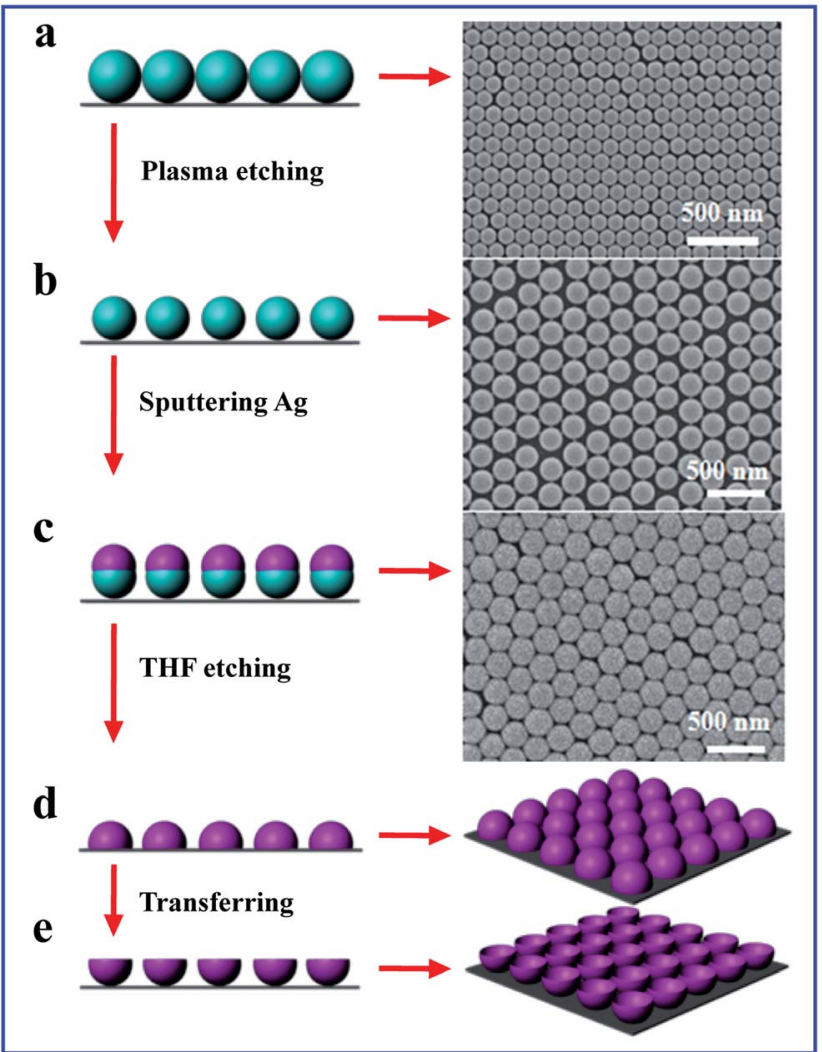

Fig. 1 Schematic and the SEM images for the fabrication process of silver nanobowl arrays. (a) Original PS array. (b) Etched PS array. (c) Ag nanocap deposited on the PS template. (d) Ag nanocap array etched by THF. (e) Ag nanobowl array transferred on a new substrate.

The period and shape of the bead array are maintained and the bead surfaces are still smooth upon etching. When the etching time is $30 \mathrm{~s}$, the neighbor beads are separated slightly, and the nanogap between adjacent PS is about $20 \mathrm{~nm}$. When the etching time is $60 \mathrm{~s}$, the neighbor beads are separated completely and the PS size is $160 \mathrm{~nm}$ with the gap around $40 \mathrm{~nm}$. When $\mathrm{Ag}$ film is deposited on the PS nanosphere arrays to form $\mathrm{Ag}$ nanocaps arrays (Fig. 1c), then the PS nanospheres were removed by immersing the samples into tetrahydrofuran (THF) solution (Fig. 1d). Ag nanobowl arrays are finally obtained by transferring the Ag film onto a new substrate as shown in Fig. 1e. Based on the close-packed and separated PS beads array, the connected and separated nanobowls arrays are fabricated.

Fig. 2 shows the SEM images of closed-packed nanobowl arrays based on PS array without etching when $80 \mathrm{~nm} \mathrm{Ag}$ film is deposited onto PS $200 \mathrm{~nm}$ templates. The diameter of the Ag nanobowl is about $200 \mathrm{~nm}$. The brims of the nanobowls are very thin and there are six notches in every nanobowl due to the shadow effect from the neighbor PS beads (Fig. 2a), as we have seen clearly in the inset. When $30 \mathrm{~s}$ etching is performed, the isolated $\mathrm{Ag}$ nanobowls with smooth brim form and no notches between adjacent bowls are observed (Fig. 2b). The tilted view of $\mathrm{Ag}$ nanobowl is given in enlarged inset of Fig. 2b, confirming the complete nanobowl shape. However, the morphology of Ag nanobowl based on the PS array etched for $60 \mathrm{~s}$ changes obviously. The nanoholes-like arrays with $200 \mathrm{~nm}$ period are observed (Fig. 2c). The film thickness and the PS diameter are almost equal when $80 \mathrm{~nm} \mathrm{Ag}$ film is deposited onto PS arrays with diameter $120 \mathrm{~nm}$ with $60 \mathrm{~nm}$ gaps. In this case, the distances between the etched PS beads are so large that the shadow effect is reduced and therefore the Ag film between the PS beads connects the nanobowls together to become procontinuous. Therefore, the nanobowl looks like holes embedded in a continuous film. Near the crack in the film, the nanobowls are observed, which confirms the nanobowl formation in the inset. Therefore, to further test the speculation, $\mathrm{Ag}$ film with the thickness of $100 \mathrm{~nm}$ is also deposited onto $200 \mathrm{~nm}$ PS beads after $60 \mathrm{~s}$ etching. The image of SEM shows the nanobowls are connected together more and the nanohole array form in which the hole size becomes smaller (Fig. 2d). The above results show the size of bowl rim and distance of neighborhood bowl can be modified easily by adjusting the PS diameter and the film thickness.

\subsection{The SERS effect for nanobowl arrays}

Fig. 3A shows the effects of different nanobowl morphologies on SERS enhancement in $\mathrm{Ag}$ nanobowl array. The main peaks located at $1614 \mathrm{~cm}^{-1}, 1589 \mathrm{~cm}^{-1}$ and $1100 \mathrm{~cm}^{-1}$, which are assigned to $\mathrm{C}-\mathrm{C}$ stretching mode, $\mathrm{C}-\mathrm{C}$ stretching mode and $\mathrm{C}-\mathrm{S}$ stretching mode, ${ }^{24}$ respectively. Compared to other samples, the largest SERS enhancement is observed on the isolated Ag nanobowl array based on PS template etched for $30 \mathrm{~s}$, the reason is that the hot-spots in the nanogaps between adjacent nanobowls generate high plasma coupling, causing the SERS enhancement. Fig. 3B clearly shows the above changing trend. For the closepacked nanobowl array (Fig. 3a), the SERS enhancement is lower than the separated nanobowl (Fig. 3b), but higher than the hole-like array (Fig. 3c and d), which is ascribed to the sharp brim of nanobowl array. For the isolated nanocaps on the etched PS, the localized surface plasmons (LSPs) form and the plasmon coupling between the neighbor nanobowls contributes to the SERS enhancement. For the hole-like array, the localized surface plasma is extended and the couplings between the neighbors are reduced due to the connection of the neighbor Ag nanobowls, which leads to the decrease of the SERS signals.

To verify the contributions of the plasmon coupling between the interior and exterior Ag layers in nanobowl structure, the nanobowl arrays of sandwich structure $\mathrm{Ag} 40 \mathrm{~nm} / \mathrm{SiO}_{2} t \mathrm{~nm} / \mathrm{Ag}$ $40 \mathrm{~nm}(t=0,1,2,3)$ are fabricated on $200 \mathrm{~nm}$ PS substrate after etching for $30 \mathrm{~s}$. The $\mathrm{SiO}_{2}$ layers with thickness from 1 to $3 \mathrm{~nm}$ are used to separate the $\mathrm{Ag}$ layer. Compared to isolated $\mathrm{Ag}$ nanobowl array without $\mathrm{SiO}_{2}$ spacer layer, Fig. 4a-d shows the SERS peaks of $\mathrm{Ag} 40 \mathrm{~nm} / \mathrm{SiO}_{2} t \mathrm{~nm} / \mathrm{Ag} 40 \mathrm{~nm}$ nanobowl $(t=0,1,2,3)$ exhibit significant SERS enhancement. The SERS enhancement reaches the maximum when $\mathrm{SiO}_{2}$ thickness is $2 \mathrm{~nm}$, which indicates that the plasma coupling occurs between interior $\mathrm{Ag}$ layer and exterior $\mathrm{Ag}$ layer through the $\mathrm{SiO}_{2}$ layer. The thin $\mathrm{SiO}_{2}$ layer is essential to transfer the strong electromagnetic field from its lower Ag layer to the upper Ag layer, ${ }^{32,33}$ which is an important factor leading to SERS enhancement. As a dielectric gap, the thin $\mathrm{SiO}_{2}$ layer could be used to tune the 


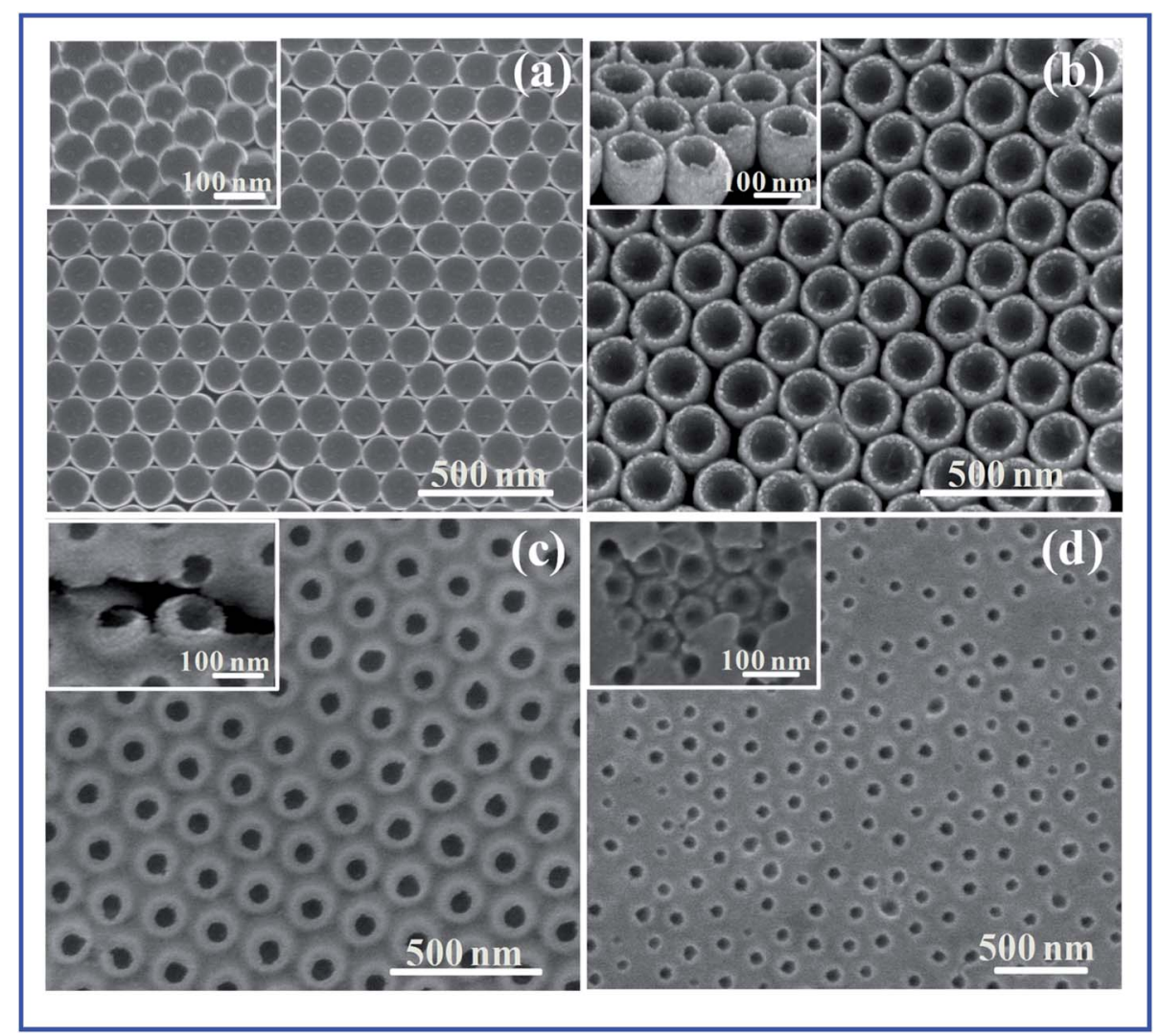

Fig. $2 \mathrm{SEM}$ images of $80 \mathrm{~nm}$ Ag nanobowl array on $\mathrm{O}_{2}$-plasma etched PS for different times (a) $0 \mathrm{~s}$, (b) $30 \mathrm{~s}$, (c) $60 \mathrm{~s}$, and (d) the $100 \mathrm{~nm}$ Ag nanobowl arrays on $\mathrm{O}_{2}$-plasma etched PS for $30 \mathrm{~s}$. The insets are cross sectional views.

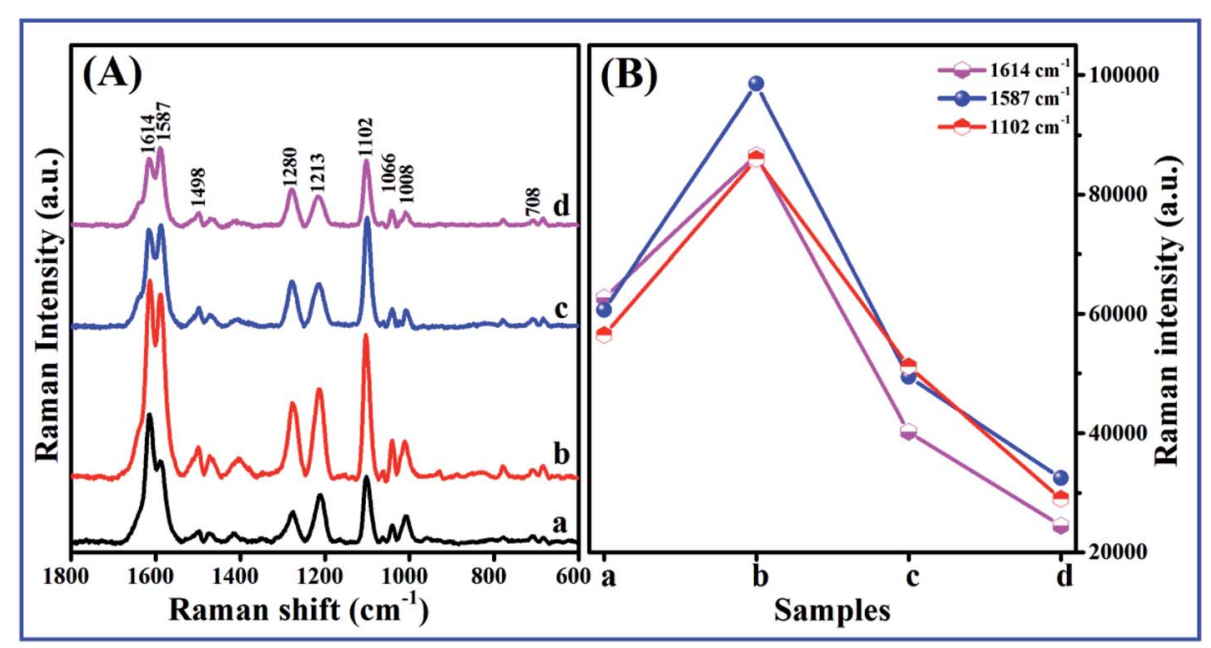

Fig. 3 (A) SERS spectra of the 4-MPy and (B) comparison of Raman intensities at $v 1614 \mathrm{~cm}^{-1}, 1587 \mathrm{~cm}^{-1}, 1102 \mathrm{~cm}^{-1}$ on $80 \mathrm{~nm}$ Ag nanobowls etched for different times: (a) $0 \mathrm{~s}$, (b) $30 \mathrm{~s}$, (c) $60 \mathrm{~s}$, (d) $100 \mathrm{~nm}$ Ag nanobowl arrays etched for $60 \mathrm{~s}$.

LSPs of the $\mathrm{Ag}$ layer in $\mathrm{Ag} / \mathrm{SiO}_{2} / \mathrm{Ag}$ nanobowls, ${ }^{34}$ resulting in optimized LSPR interaction.

To verify the general applicability of the fabricated samples as the SERS substrates, we immersed the $\mathrm{Ag} 40 \mathrm{~nm} / \mathrm{SiO}_{2} 2 \mathrm{~nm} /$ Ag $40 \mathrm{~nm}$ arrays $(t=0,1,2$, and 3$)$ in an ethanol solution of $10^{-6} \mathrm{M}$ R6G for $1 \mathrm{~h}$. Raman spectra of the surfaces were collected with the same methods as above. Fig. 5 shows the characteristic Raman signals of R6G molecules with different samples: (a) $\mathrm{Ag} 80 \mathrm{~nm}$ nanobowl array without etched, $\mathrm{Ag} 40$ $\mathrm{nm} / \mathrm{SiO}_{2} t \mathrm{~nm} / \mathrm{Ag} 40 \mathrm{~nm}$ nanobowl array with different thickness of $\mathrm{SiO}_{2}$ film: (b) 1, (c) 2, and (d) 3. All the Raman bands match well with those of the characteristic spectrum of R6G as followings. ${ }^{35}$ The peaks of 1650, 1508, 1363, 1188, 773 and $612 \mathrm{~cm}^{-1}$ are attributed to $\mathrm{C}-\mathrm{H}$ in-plane bending, $\mathrm{C}-\mathrm{O}-\mathrm{C}$ 


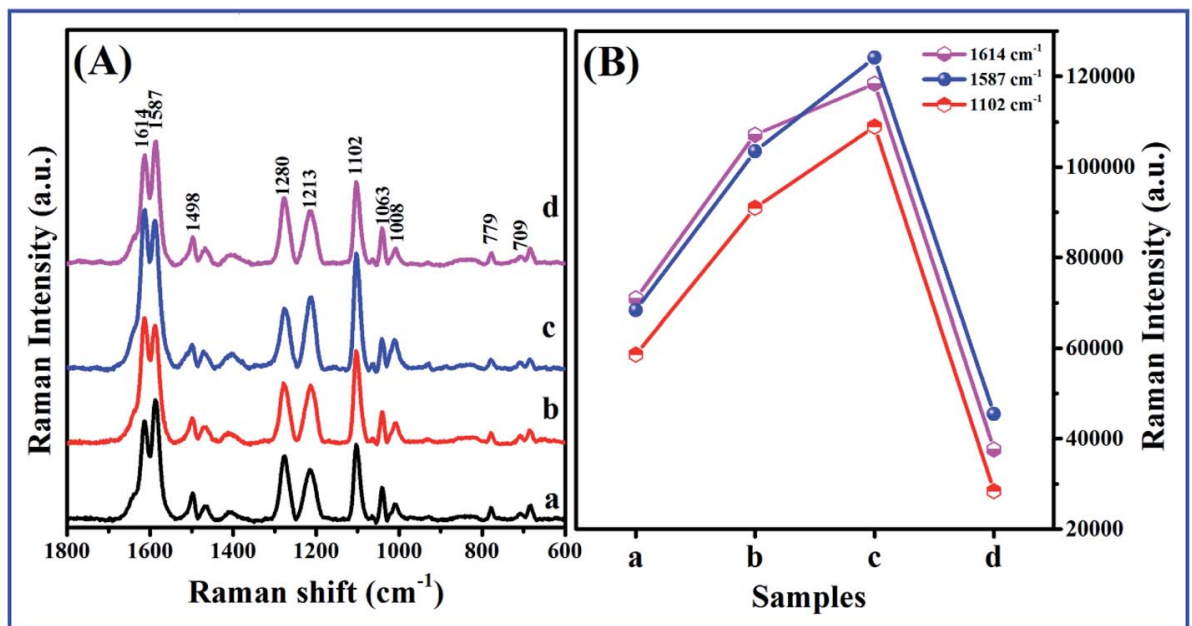

Fig. 4 (A) SERS spectra of the 4-MPy and (B) comparison of Raman intensities at $1614 \mathrm{~cm}^{-1}, 1587 \mathrm{~cm}^{-1}, 1102 \mathrm{~cm}^{-1}$ obtained on different samples: (a) Ag $80 \mathrm{~nm}$ nanobowl array without etched, $\mathrm{Ag} 40 \mathrm{~nm} / \mathrm{SiO}_{2} \mathrm{tnm} / \mathrm{Ag} 40 \mathrm{~nm}$ nanobowl array with different thickness of SiO ${ }_{2}$ film: (b) 1 , (c) 2 , (d) 3 .

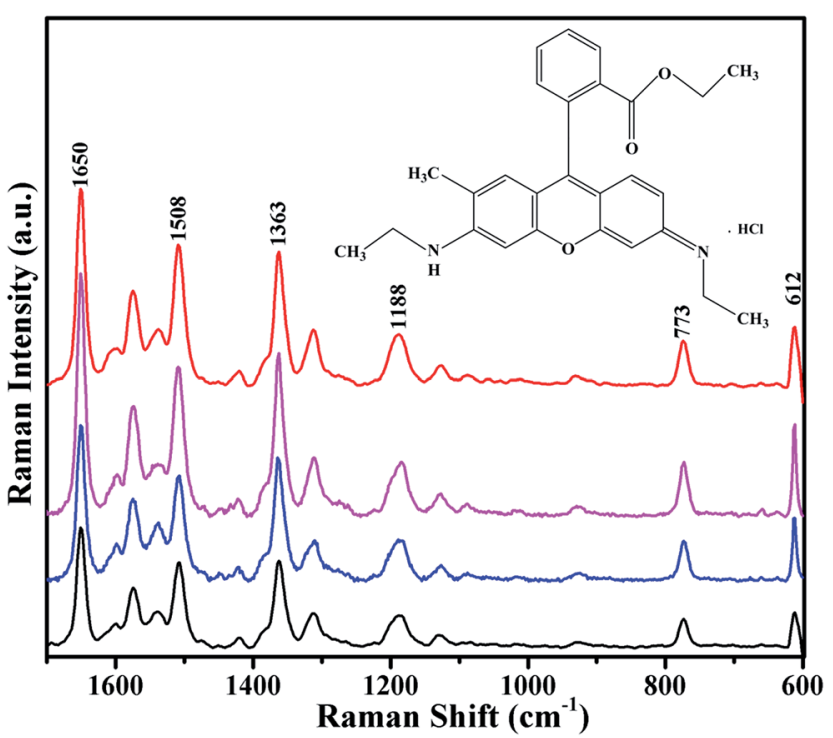

Fig. 5 SERS spectra of different samples: (a) Ag $80 \mathrm{~nm}$ nanobowl array without etched, $\mathrm{Ag} 40 \mathrm{~nm} / \mathrm{SiO}_{2} \mathrm{tnm} / \mathrm{Ag} 40 \mathrm{~nm}$ nanobowl array with different thickness of $\mathrm{SiO}_{2}$ film: (b) 1, (c) 2, and (d) 3 .

stretching, C-C stretching of the aromatic ring, C-H in-plane bending, $\mathrm{C}-\mathrm{H}$ out-of-plane bending and $\mathrm{C}-\mathrm{C}-\mathrm{C}$ in-plane bending modes, respectively. ${ }^{36,37}$ Compared to the peak intensities between different substrates, significantly, the highest Raman peak intensities can be clearly observed for the $\mathrm{Ag} 40$ $\mathrm{nm} / \mathrm{SiO}_{2} 2 \mathrm{~nm} / \mathrm{Ag} 40 \mathrm{~nm}$ arrays (Fig. 5c). Combining the above SERS spectra (Fig. 4) and this experimental SERS result, which further demonstrates the $\mathrm{Ag} 40 \mathrm{~nm} / \mathrm{SiO}_{2} 2 \mathrm{~nm} / \mathrm{Ag} 40 \mathrm{~nm}$ arrays have good SERS performance.

For any new SERS substrates, the reproducibility of Raman signals is an important factor. Fig. 6 shows the SERS spectra of the $\mathrm{Ag} 40 \mathrm{~nm} / \mathrm{SiO}_{2} 2 \mathrm{~nm} / \mathrm{Ag} 40 \mathrm{~nm}$ arrays, which was collected from 10 random positions of the substrates. The relative

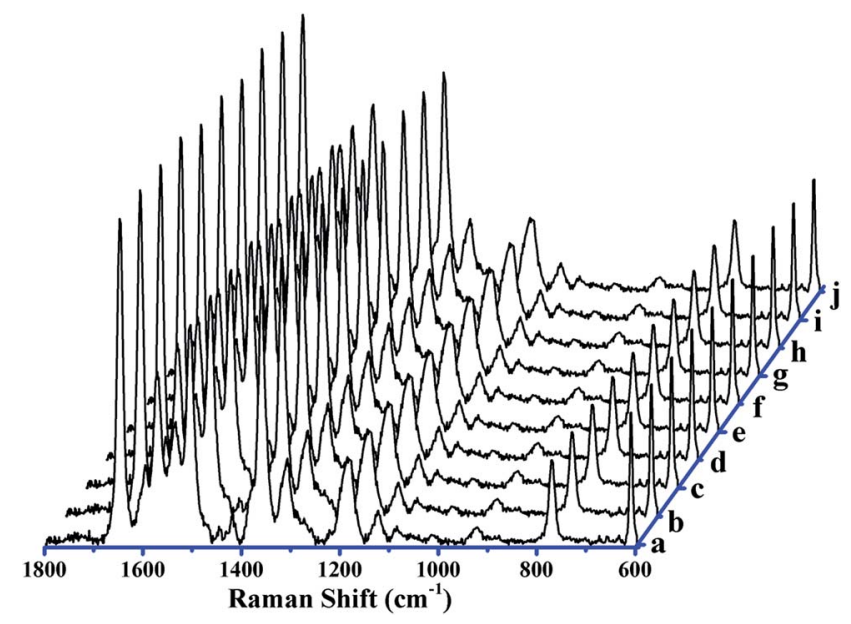

Fig. 6 The reproducibility of SERS spectra for the Ag $40 \mathrm{~nm} / \mathrm{SiO}_{2} 2$ $\mathrm{nm} / \mathrm{Ag} 40 \mathrm{~nm}$ nanobowl array.

standard deviation (RSD) of SERS intensity at the band of the $1650 \mathrm{~cm}^{-1}$ reaches to less than $6.4 \%$, indicating the good reproducibility of the SERS substrates.

\subsection{Characterization of nanobowl arrays}

To further verify the chemical compositions of films, XPS measurements for the $\mathrm{Ag} 40 \mathrm{~nm} / \mathrm{SiO}_{2} 2 \mathrm{~nm} / \mathrm{Ag} 40 \mathrm{~nm}$ arrays were performed. The XPS spectra of the full-scan spectrum, Ag 3d, Si 2p, O 1s and C 1s from films are shown in Fig. 7A-E, respectively. Fig. 7A shows that the full-scan XPS spectrum (0$1000 \mathrm{eV}$ ) of $\mathrm{Ag} / \mathrm{SiO}_{2} / \mathrm{Ag}$ films, the peaks of $\mathrm{Ag}, \mathrm{Si}, \mathrm{C}$ and $\mathrm{O}$ have appeared. It is important to point out that the presence of $\mathrm{C} 1 \mathrm{~s}$ $(284.68 \mathrm{eV})$ may be due to the carbon tape used during the measurements, which is generally used as an internal reference in the spectra (Fig. 7E). Fig. 7B depicts that the two distinct peaks for $\mathrm{Ag}$ correspond to the $\mathrm{Ag} 3 \mathrm{~d}_{5 / 2}$ and $\mathrm{Ag} 3 \mathrm{~d}_{3 / 2}$ orbitals, 

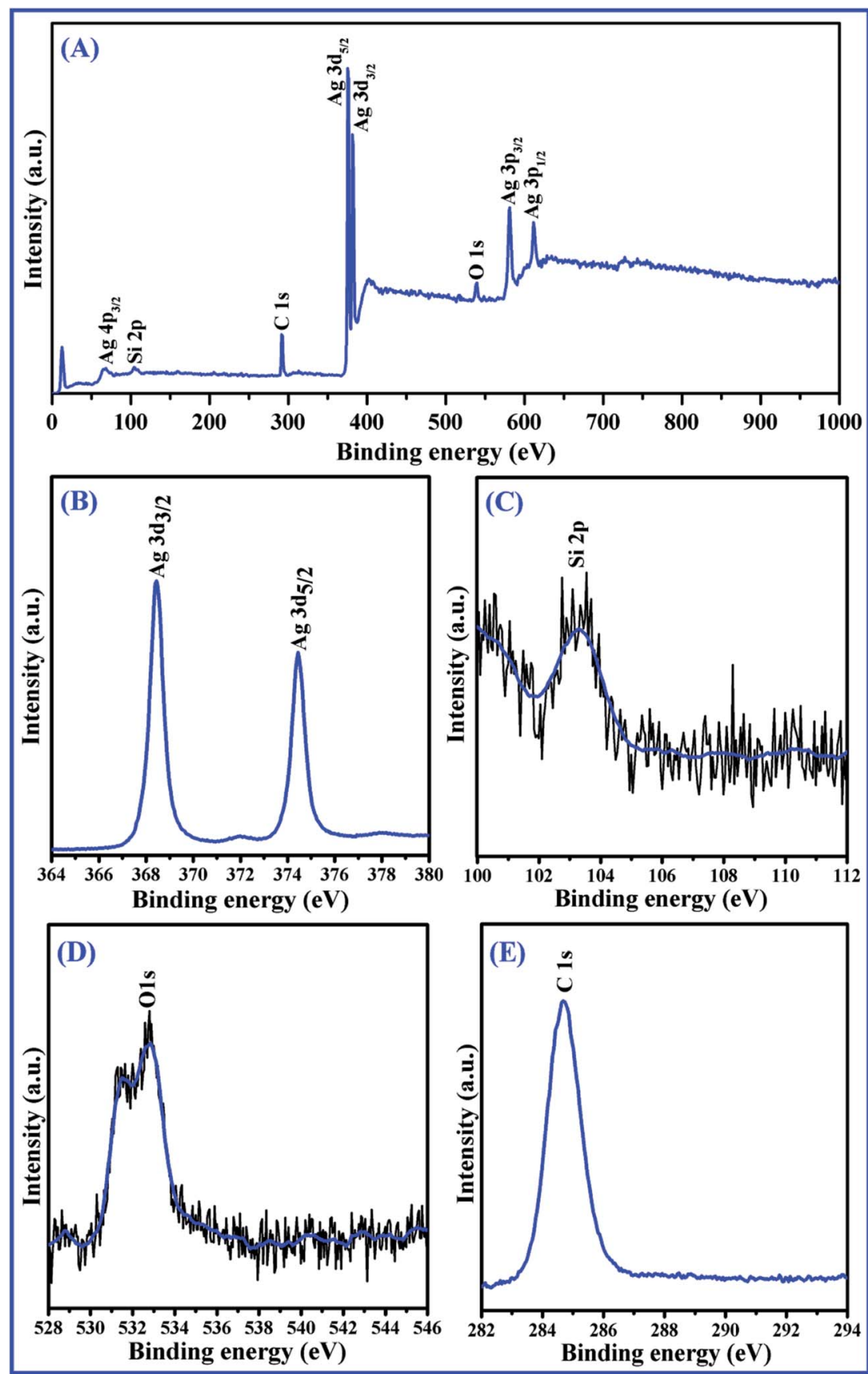

Fig. 7 The XPS spectra of (A) the full-scan spectrum, (B) Ag 3d, (C) Si 2p, (D) O 1s, and (E) C 1s of Ag $40 \mathrm{~nm} / \mathrm{SiO}_{2} 2 \mathrm{~nm} / \mathrm{Ag} 40 \mathrm{~nm}$ nanobowl arrays.

which possess a doublet laid on $368.46 \mathrm{eV}$ and $374.46 \mathrm{eV}$, respectively. The distinct peak of $\mathrm{Si} 2 \mathrm{p}$ locate at $103.3 \mathrm{eV}$ was shown Fig. 7C. No evidence of the single Si peak was observed, which confirmed that $\mathrm{Si}$ exit only in the oxidized state $\mathrm{SiO}_{2}$ rather than from Si substrates. Fig. 7D shows the O 1s XPS spectrum of the sample, which can be deconvolved into the two strong peaks, indicating the existence of two different oxygen environments. ${ }^{38,39}$ The higher binding energy at $532.81 \mathrm{eV}$ originates from the $\mathrm{Si}-\mathrm{O}$ bond formation, one peak with a lower binding energy of $531.49 \mathrm{eV}$ derives from the surface adsorbed oxygen, which is possibly attributed to environmental moisture trapped on the film surface.

\subsection{SERS enhancement factor of $\mathrm{Ag}$ and $\mathrm{Ag} / \mathrm{SiO}_{2} / \mathrm{Ag}$} nanobowl arrays

The EF is defined as EF $=\left(I_{\text {SERS }} \times N_{\text {vol }}\right)\left(I_{\text {RS }} \times N_{\text {surf }}\right),{ }^{\mathbf{4 0 - 4 2}}$ where $I_{\mathrm{SERS}}$ and $I_{\mathrm{RS}}$ are the intensities of the band $1614 \mathrm{~cm}^{-1}$ from the SERS substrates and reference solution $\left(1 \times 10^{-3} \mathrm{M}\right)$, respectively. $N_{\mathrm{vol}}=C_{\mathrm{RS}} V$ is the average number of molecules in the scattering volume $(V)$ for the Raman (non-SERS) measurement and $N_{\text {surf }}$ is the average number of adsorbed molecules in the scattering volume for the SERS experiments. The 4-MPy forms a homogeneous monolayer on the substrate after absorption (Fig. $\mathrm{S} 4 \dagger$ ), and the surface area occupied by single 4-Mpy 


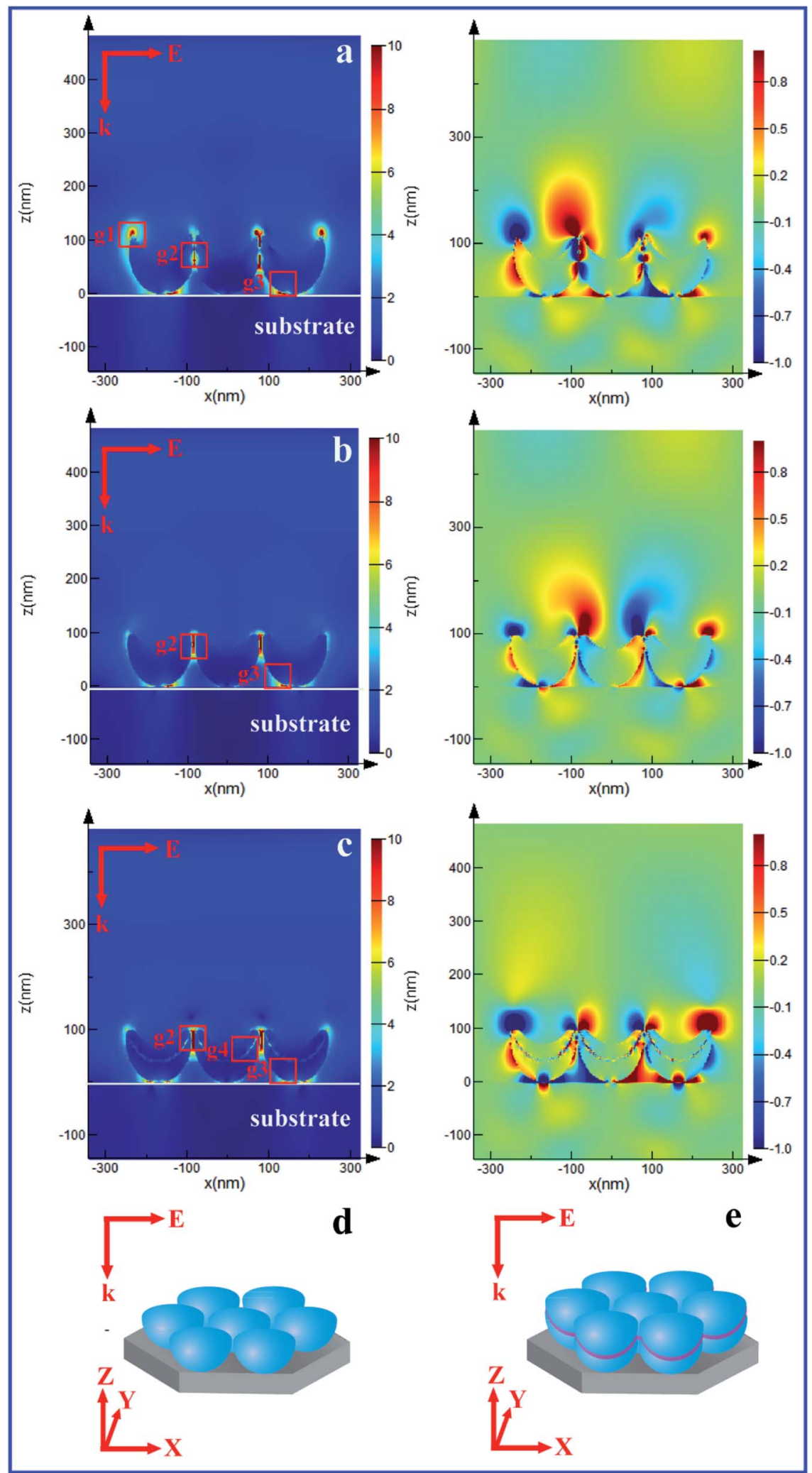

Fig. 8 Electric field distribution in left column and charge density distribution in right column for different nanobowls excited at $514.5 \mathrm{~nm}$ laser wavelength. (a) Close-packed Ag $80 \mathrm{~nm}$ nanobowl array etched for $0 \mathrm{~s}$. (b) Separated Ag $80 \mathrm{~nm}$ nanobowl array etched for $30 \mathrm{~s}$. (c) Separated Ag $40 \mathrm{~nm} / \mathrm{SiO}_{2} 2 \mathrm{~nm} / \mathrm{Ag} 40 \mathrm{~nm}$ nanobowls etched for $30 \mathrm{~s}$. The color bar represents the value of $|E| /\left|E_{\mathrm{o}}\right|$. (d) The simulation model image of Ag nanobowl array. (e) The simulation model image of $\mathrm{Ag} / \mathrm{SiO}_{2} / \mathrm{Ag}$ nanobowl array. 
molecule on the silver substrate value is estimated to be 0.30 $\mathrm{nm}^{2} .^{2,43,44}$ The laser spot is a circle with a diameter of $1 \mu \mathrm{m} . N_{\text {surf }}$ can be calculated to be $2 \times 10^{5}$. For the optical configuration and microscope employed, the effective focused depth is $1 \mu \mathrm{m}$. $N_{\mathrm{vol}}=C_{\mathrm{RS}} V$ is about $4.7 \times 10^{9}$. So the $\mathrm{EF}$ is calculated to be 1.37 $\times 10^{7}$ and $8 \times 10^{7}$ for $\mathrm{Ag} / \mathrm{SiO}_{2} / \mathrm{Ag}$ nanobowl arrays when $\mathrm{SiO}_{2}$ thickness is $1 \mathrm{~nm}$ and $2 \mathrm{~nm}$, and $7.5 \times 10^{5}$ for $\mathrm{Ag}(80 \mathrm{~nm})$ nanobowl and $2.8 \times 10^{6}$ when $\mathrm{SiO}_{2}$ thickness is $3 \mathrm{~nm}$.

\subsection{FDTD solutions}

The SERS enhancement was explained from the electromagnetic field distribution and charge density distribution for the different nanobowl arrays, as shown in Fig. 7. The local field enhancement of the vertical cross sections of the nanobowls with $\mathrm{Ag}$ thickness $80 \mathrm{~nm}$ and $\mathrm{Ag} 40 \mathrm{~nm} / \mathrm{SiO}_{2} 2 \mathrm{~nm} / \mathrm{Ag} 40 \mathrm{~nm}$ nanobowls on silicon substrate was simulated by FDTD solutions. All plots of the data are shown for the $x-z$ vertical cross sections containing the center of the nanobowl arrays. The simulation models of $\mathrm{Ag}$ and $\mathrm{Ag} / \mathrm{SiO}_{2} / \mathrm{Ag}$ nanobowl arrays are shown in Fig. $8 \mathrm{~d}$ and e, respectively. Fig. 8a shows that the resonances for nanobowls corresponding to the close-packed nanobowls with $\mathrm{Ag}$ thickness $80 \mathrm{~nm}$, as we can see, the enhanced field is ascribed to the plasmon couplings between vertexes of the Ag nanobowl arrays (g1), the gap plasmons at the crevice nanogap regions (2 $\mathrm{nm})(\mathrm{g} 2)$ and the LSPs between $\mathrm{Ag}$ nanobowl and the substrate (g3). The charge density distribution plots (in right column) show distinct SPR between Ag nanobowls, which confirms that the resonance belongs to quadrupole resonance mode in essence. ${ }^{45}$ Fig. 8b shows that the resonances for the separated Ag nanobowls, and the strongly enhanced local field is confined to the very narrow nanogap regions at the separated $\mathrm{Ag}$ nanobowl arrays (g2) and the LSPs between Ag nanobowl and the substrate (g3). The charge distribution is supporting a symmetrical dipole resonance mode. The resonances for the $\mathrm{Ag} 40 \mathrm{~nm} / \mathrm{SiO}_{2} 2 \mathrm{~nm} / \mathrm{Ag}$ $40 \mathrm{~nm}$ nanobowls were shown in Fig. 8c. From the point of the Efield distribution, we think that the field enhancements can be mainly attributed to the SPPs of interparticles that propagate along the $\mathrm{Ag}-\mathrm{SiO}_{2}$ interfaces $(\mathrm{g} 4){ }^{46}$ in addition to the contributions of the gaps between $\mathrm{Ag} / \mathrm{SiO}_{2} / \mathrm{Ag}$ nanobowls $(\mathrm{g} 2)$ and the LSPs between Ag nanobowl and the substrate (g3). By comparing the FDTD simulation of the Ag nanobowl arrays (Fig. 8b), the electromagnetic field experiences a change, resulting in an extra distribution of the electromagnetic field between the Ag layer and $\mathrm{Ag}$ layer. The presence of the dielectric $\mathrm{SiO}_{2}$ layer provides an additional field confinement in the hot spot for the case of the new, quadrupolar plasmon mode. ${ }^{47}$ Strong SPR between $\mathrm{Ag} / \mathrm{SiO}_{2} /$ $\mathrm{Ag}$ nanobowls confirms that the resonance belongs to symmetrical dipole resonance mode. It is clear that adding insulator $\mathrm{SiO}_{2}$ layer can increase the substrate's SERS activity, and there exists an optimal $\mathrm{SiO}_{2}$ thickness for the maximal Raman enhancement.

\section{Conclusions}

In conclusion, the nanobowls or nanohole-like arrays of $\mathrm{Ag}$ and $\mathrm{Ag} / \mathrm{SiO}_{2} / \mathrm{Ag}$ with different morphologies are successfully fabricated by the combination of NSL technology and LBL film deposition. The morphologies are modified by changing the film thickness and the size of PS beads. In contrast to the nanohole-like array, the nanobowl array exhibits stronger SERS signal, which is ascribed to two important factors. One is that the gaps between adjacent PS or sharp edges around rims contribute to the SERS enhancement. The other is that the plasma coupling at the bowl bottom gaps between the interior and exterior surface of nanobowl structure also contributes to the high SERS signal, as verified by $\mathrm{Ag} / \mathrm{SiO}_{2} / \mathrm{Ag}$ nanobowl structure.

\section{Conflicts of interest}

There are no conflicts to declare.

\section{Acknowledgements}

This work is supported by the National Natural Science Foundation of China (No. 61575080, 61675090 and 21676115), National Youth Program Foundation of China (No. 21546013, 61405072 and 51609100), Program for the development of Science and Technology of Jilin province (No. 20150519024JH, $20150520015 \mathrm{JH}, 20160101287 \mathrm{JC}$ and 20140519003JH), and Technology of Education Department of Jilin Province (No. 2016-217).

\section{References}

1 M. Fleischmann, P. J. Hendra and A. J. McQuillan, Ramanspectra of pyridine adsorbed at silver electrode, Chem. Phys. Lett., 1974, 26, 163-166.

2 J. Chen, B. Shen, G. Qin, X. Hu, L. Qian, Z. Wang, S. Li, Y. Ren and L. Zuo, Fabrication of large-area, high-enhancement SERS substrates with tunable interparticle spacing and application in identifying microorganisms at the single cell level, J. Phys. Chem. C, 2012, 116, 3320-3328.

3 M. Bechelany, P. Brodard, J. Elias, A. Brioude, J. Michler and L. Philippe, Simple synthetic route for SERS-active gold nanoparticles substrate with controlled shape and organization, Langmuir, 2010, 26, 14364-14371.

4 J. Theiss, P. Pavaskar, P. M. Echternach, R. E. Muller and S. B. Cronin, Plasmonic nanoparticle arrays with nanometer separation for high-performance SERS substrates, Nano Lett., 2010, 10, 2749-2754.

5 A. Barhoumi and N. J. Halas, Label-free detection of DNA hybridization using surface enhanced Raman spectroscopy, J. Am. Chem. Soc., 2010, 132, 12792-12793.

6 S. L. Kleinman, E. Ringe, N. Valley, K. Wustholz, E. Phillips, K. Scheidt, G. Schata and R. P. Van Duyne, Single-molecule surface-enhanced Raman spectroscopy of crystal violet isotopologues: theory and experiment, J. Am. Chem. Soc., 2011, 133, 4115-4122.

7 L. Chen, H. H. Sun, Y. Zhao, Y. J. Zhang, Y. X. Wang, L. Yang, X. L. Zhang, Y. H. Jiang, Z. Hua and J. H. Yang, Plasmonicinduced SERS enhancement of shell-dependent Ag@Cu2O core-shell nanoparticles, $R S C$ Adv., 2017, 7, 16553-16560. 
8 L. Chen, Y. Zhao, Y. J. Zhang, M. M. Liu, Y. X. Wang, X. Qu, L. Yang, J. Li, X. Y. Liu and J. H. Yang, Design of $\mathrm{Cu}_{2} \mathrm{O}-\mathrm{Au}$ composite microstructures for surface-enhanced Raman scattering study, Colloids Surf., A, 2016, 507, 96102.

9 K. D. Alexander, K. Skinner, S. Zhang, H. Wei and R. Lopez, Tunable SERS in gold nanorod dimers through strain control on an elastomeric substrate, Nano Lett., 2010, 10, 4488-4493.

10 M. A. Noginov, G. Zhu, A. M. Belgrave, R. Bakker, V. M. Shalaev, E. E. Narimanov, S. Stout, E. Herz, T. Suteewong and U. Wiesner, Demonstration of a spaserbased nanolaser, Nature, 2009, 460, 1110-1112.

11 L. S. Slaughter, Y. P. Wu, B. A. Willingham, P. Nordlander and S. Link, Effects of symmetry breaking and conductive contact on the plasmon coupling in gold nanorod dimmers, ACS Nano, 2010, 4, 4657-4666.

12 T. Liu, D. S. Li, Y. Zou, D. R. Yang, H. L. Li, Y. M. Wu and M. H. Jiang, Preparation of metal @ silica core-shell particle films by interfacial self-assembly, $J$. Colloid Interface Sci., 2010, 350, 58-62.

13 Q. Fu, Z. B. Zhan, J. X. Dou, X. Z. Zheng, R. Xu, M. H. Wu and Y. Lei, Highly reproducible and sensitive SERS substrates with $\mathrm{Ag}$ inter-nanoparticle gaps of $5 \mathrm{~nm}$ fabricated by ultrathin aluminum mask technique, ACS Appl. Mater. Interfaces, 2015, 7, 13322-13328.

14 H. Lange, B. H. Juárez, A. Carl, M. Richter, N. G. Bastús, H. Weller, C. Thomsen, R. V. Klitzing and A. Konrr, Tunable plasmon coupling in distance-controlled gold nanoparticles, Langmuir, 2012, 28, 8862-8866.

15 G. Q. Liu, Y. Li, G. T. Duan, J. J. Wang, C. H. Liang and W. P. Cai, Tunable surface plasmon resonance and strong SERS performances of $\mathrm{Au}$ opening-nanoshell ordered arrays, ACS Appl. Mater. Interfaces, 2011, 4, 1-5.

16 L. Gunnarsson, E. J. Bjerneld, H. Xu, S. Petronis, B. Kasemo and $\mathrm{M}$. Kall, Interparticle coupling effects in nanofabricated substrates for surface-enhanced Raman scattering, Appl. Phys. Lett., 2001, 78, 802-804.

17 A. Gopinath, S. V. Boriskina, W. R. Premasiri, L. Ziegler, B. M. Reinhard and L. D. Negro, Plasmonic nanogalaxies: multiscale aperiodic arrays for surface-enhanced Raman sensing, Nano Lett., 2009, 9, 3922-3929.

18 S. Y. Chou, P. P. Krauss and P. J. Renstrom, Nanoimprint lithography, J. Vac. Sci. Technol., B: Microelectron. Nanometer Struct., 1996, 14, 4129-4133.

19 Y. X. Wang, X. Y. Zhao, L. Chen, S. Chen, M. B. Wei, M. Gao, Y. Zhao, C. Wang, X. Qu, Y. J. Zhang and J. H. Yang, Ordered nanocap array composed of $\mathrm{SiO}_{2}$-isolated $\mathrm{Ag}$ islands as SERS platform, Langmuir, 2014, 30, 15285-15291.

20 K. Chen, B. B. Rajeeva, Z. L. Wu, M. Rukavina, T. D. Dao, S. Ishii, M. Aono, T. Nagao and Y. B. Zheng, Moiré nanosphere lithography, ACS Nano, 2015, 9, 6031-6040.

21 J. Yu, C. Geng, L. Zheng, Z. H. Ma, T. Y. Tan, X. Q. Wang, Q. F. Yan and D. Z. Shen, Preparation of high-quality colloidal mask for nanosphere lithography by a combination of air/water interface self-assembly and solvent vapor annealing, Langmuir, 2012, 28, 1268112689.
22 A. B. Zrimsek, A. I. Henry and R. P. Van Duyne, Single molecule surface-enhanced Raman spectroscopy without nanogaps, J. Phys. Chem. Lett., 2013, 4, 3206-3210.

23 L. Ji, Y. F. Chang, B. Fowler, Y. C. Chen, T. M. Tsai, K. C. Chang, M. C. Chen, T. C. Chang, S. M. Sze, E. T. Yu and J. C. Lee, Integrated one diode-one resistor architecture in nanopillar SiOx resistive switching memory by nanosphere lithography, Nano Lett., 2013, 14, 813-818.

24 Y. X. Wang, X. Y. Zhao, W. T. Gao, L. Chen, S. Chen, M. B. Wei and M. Gao, Au/Ag bimetal nanogap arrays with tunable morphologies for surface-enhanced Raman scattering, RSC Adv., 2015, 5, 7454-7460.

25 C. X. Wang, W. D. Ruan, N. Ji, W. Ji, S. Lv, C. Zhao and B. Zhao, Preparation of nanoscale Ag semishell array with tunable interparticle distance and its application in surface-enhanced Raman scattering, J. Phys. Chem. C, 2010, 114, 2886-2890.

26 X. Y. Zhao, J. H. Wen, M. N. Zhang, D. H. Wang, Y. X. Wang, L. Chen, Y. J. Zhang, J. H. Yang and Y. W. Du, Design of Hybrid Nanostructural Arrays to Manipulate SERS-Active Substrates by Nanosphere Lithography, ACS Appl. Mater. Interfaces, 2017, 9, 7710-7716.

27 T. Y. Jeon, S. G. Park, S. Y. Lee, H. C. Jeon and S. M. Yang, Shape control of $\mathrm{Ag}$ nanostructures for practical SERS substrates, ACS Appl. Mater. Interfaces, 2013, 5, 243-248.

28 D. D. Lin, Z. L. Wu, S. J. Li, W. Q. Zhao, C. J. Ma, J. Wang, Z. M. Jiang, Z. Y. Zhong, Y. B. Zheng and X. J. Yang, LargeArea Au-Nanoparticle-Functionalized Si Nanorod Arrays for Spatially Uniform Surface-Enhanced Raman Spectroscopy, ACS Nano, 2017, 11, 1478-1487.

29 L. Chen, H. H. Sun, Y. Zhao, R. X. Gao, Y. X. Wang, Y. Liu, Y. J. Zhang, Z. Hua and J. H. Yang, Iron Layer-Dependent Surface-Enhanced Raman Scattering of Hierarchical Nanocap Arrays, Appl. Surf. Sci., 2017, 423, 1124-1133.

30 Y. J. Zhang, C. Wang, J. Wang, J. P. Wang, L. Chen, J. Li, Y. Liu, X. Y. Zhao, Y. X. Wang and J. H. Yang, Nanocap array of Au: Ag composite for surface-enhanced Raman scattering, Spectrochim. Acta, Part A, 2016, 152, 461-467.

31 S. Siddhanta, V. Thakur, C. Narayana and S. M. Shivaprasad, Universal metal- semiconductor hybrid nanostructured SERS substrate for biosensing, ACS Appl. Mater. Interfaces, 2012, 4, 5807-5812.

32 J. F. Li, Y. F. Huang, Y. Ding, Z. L. Yang, S. B. Li, X. S. Zhou, F. R. Fan, W. Zhang, Z. Y. Zhi, D. Y. Wu, B. Ren, Z. L. Wang and Z. Q. Tian, Shell-isolated nanoparticle-enhanced Raman spectroscopy, Nature, 2010, 464, 392-395.

$33 \mathrm{H}$. Miyazaki and Y. Kurokawa, Squeezing visible light waves into a 3-nm-thick and 55-nm-long plasmon cavity, Phys. Rev. Lett., 2006, 96, 097401.

34 T. K. Liu, M. S. Tsai, W. C. Hung, C. T. Kuo, D. P. Wang and I. M. Jiang, Field-enhanced Raman scattering by silver nanoparticle with graded $\mathrm{SiO}_{2}$ coating, Appl. Phys. Lett., 2013, 102, 153105.

35 P. Hildebrandt and M. Stockburger, Surface-enhanced resonance Raman spectroscopy of Rhodamine 6G adsorbed on colloidal silver, J. Phys. Chem., 1984, 88, 59355944 . 
36 M. Liu, L. Sun, C. W. Cheng, H. L. Hu, Z. X. Shen and H. J. Fan, Highly effective SERS substrates based on an atomic-layer-deposition-tailored nanorod array scaffold, Nanoscale, 2011, 3, 3627-3630.

37 C. Y. Niu, B. F. Zou, Y. Q. Wang, C. Lin, H. H. Zheng and S. M. Zhou, Highly Sensitive and Reproducible SERS Performance from Uniform Film Assembled by Magnetic Noble Metal Composite Microspheres, Langmuir, 2016, 32, 858-863.

38 M. Chen, X. Wang, Y. H. Yu, Z. L. Pei, X. D. Bei, C. Sun, R. F. Huang and L. S. Wen, X-ray photoelectron spectroscopy and auger electron spectroscopy studies of Aldoped ZnO films, Appl. Surf. Sci., 2000, 158, 134-140.

39 Z. Hua, Y. P. Song, Y. R. Sui, Y. J. Wu, S. Q. Lv, B. Yao, L. Xiao, Z. W. Wang and X. Y. Liu, Effect of $\mathrm{Ar} / \mathrm{O}_{2}$ gas ratios on the structure, electrical and optical properties of Na-doped ZnCdO film synthesized by magnetron sputtering, J. Mater. Sci.: Mater. Electron., 2016, 27, 8745-8752.

40 Y. L. Wang, X. Q. Zou, W. D. Ren, W. D. Wang and E. Wang, Effect of silver nanoplates on Raman spectra of paminothiophenol assembled on smooth macroscopic gold and silver surface, J. Phys. Chem. C, 2007, 111, 3259-3265.

41 B. Ren, X. F. Lin, Z. L. Yang, G. K. Liu, R. F. Aroca, B. W. Mao and Z. Q. Tian, Surface-enhanced Raman scattering in the ultraviolet spectral region: UV-SERS on rhodium and ruthenium electrodes, J. Am. Chem. Soc., 2003, 125, 9598-9599.
42 S. M. Zhu, C. Z. Fan, J. Q. Wang, J. N. He, E. Liang and M. J. Chao, Surface enhanced Raman scattering of 4aminothiophenol sandwiched between $\mathrm{Ag}$ nanocubes and smooth Pt substrate: The effect of the thickness of Pt film, J. Appl. Phys., 2014, 116, 044312.

43 T. V. Teperik, V. V. Popov and F. J. De Abajo, Void plasmons and total absorption of light in nanoporous metallic films, Phys. Rev. B: Condens. Matter Mater. Phys., 2005, 71, 085408.

44 W. B. Cai, B. Ren, X. Q. Li, C. X. She, F. M. Liu, X. W. Cai and Z. Q. Tian, Investigation of surface-enhanced Raman scattering from platinum electrodes using a confocal Raman microscope: dependence of surface roughening pretreatment, Surf. Sci., 1998, 406, 9-22.

45 M. Frederiksen, V. E. Bochenkov, M. B. Cortie and D. S. Sutherland, Plasmon hybridization and field confinement in multilayer metal-dielectric nanocups, $J$. Phys. Chem. C, 2013, 117, 15782-15789.

46 J. Lee, Q. P. Zhang, S. Y. Park, A. Choe, Z. Y. Fan and H. Ko, Particle-film plasmons on periodic silver film over nanosphere (AgFON): a hybrid plasmonic nanoarchitecture for surface-enhanced Raman spectroscopy, ACS Appl. Mater. Interfaces, 2015, 8, 634-642.

47 F. F. Wen, J. Ye, N. Liu, P. V. Dorpe, P. Nordlander and N. J. Halas, Plasmon transmutation: inducing new modes in nanoclusters by adding dielectric nanoparticles, Nano Lett., 2012, 12, 5020-5026. 